\title{
The Energy of a Trapped Interacting Bose Gas
}

\author{
Hualin Shi and Wei-Mou Zheng \\ Institute of Theoretical Physics, Academia Sinica, Beijing 100080,China
}

\begin{abstract}
A Bose gas in an external potential is studied by means of the semi-classical approximation. Analytical results are derived for the energy of an interacting Bose gas in a generic power-law trapping potential. An expression for the chemical potential below the critical temperature is also obtained. The theoretical results are in qualitative agreement with a recent energy measurement.
\end{abstract}

PACS numbers: 03.75.Fi, 32.80.Pj

The condensation of an ideal Bose-Einstein gas is one of the most striking consequences of quantum statistics [1]. When Bose-Einstein condensation (BEC) occurs at a sufficiently low temperature, the zero momentum state can become macroscopically occupied. For many years, it was considered hopeless to experimentally observe BEC in an atomic gas with weak interactions. With the development of techniques to trap and cool atoms, BEC was recently observed directly in dilute atomic vapors 3 -5. The new experimental achievements have stimulated great interest in the theoretical study of inhomogeneous Bose gases.

The thermodynamic properties of trapped atomic Bose gases undergoing BEC can be altered by the spatially varying trapping potential. The interaction between atoms may have a significant effect on the thermodynamic properties. There have been several investigations analyzing the dependence of the critical temperature on the trapping potential and weak interaction in the Bose gas [6 8]. The thermodynamic properties of Bose gases in an external potential have also been discussed in Refs. [6,9].

In a recent experiment [10], after turning off the trapping field, the kinetic energy of a sufficiently expanded atom cloud was measured. The experimental data is in a good agreement with the theory of a trapped ideal Bose gas when the temperature is above critical temperature. However, a discrepancy with the theory of ideal Bose gases is found below the critical temperature. To explain this discrepancy it is important to know how the mutual interaction affects the energy. Here we shall derive some analytical expressions for the energy of a trapped non-interacting and interacting Bose gas under the semi-classical approximation.

First, let us calculate the kinetic and potential energies of an ideal Maxwell-Boltzmann gas and of an ideal BoseEinstein gas in an external potential. For an ideal Maxwell-Boltzmann gas, according to the Maxwell-Boltzmann distribution, the local atom number density is given by

$$
n_{\mathrm{MB}}(\mathbf{r}, \mathbf{p}, T)=\exp \left[-\beta\left(p^{2} / 2 m+V(\mathbf{r})-\mu\right)\right]
$$

where $\beta=k T$ and $\mu$ is the chemical potential which comes from the normalization. Integration of the number density (1) over the whole phase space gives the total number of atoms

$$
N=\frac{1}{h^{3}} \int n_{\mathrm{MB}}(\mathbf{r}, \mathbf{p}, T) d \mathbf{p} d \mathbf{r}=\frac{z}{\lambda^{3}} \int \exp [-\beta V(\mathbf{r})] d \mathbf{r}
$$

where

$$
\lambda=\left(\frac{2 \pi \hbar^{2}}{m k T}\right)^{1 / 2}, \quad z=e^{\beta \mu}
$$

For the generic power-law potential discussed in Ref. [6]

$$
V(\mathbf{r})=\epsilon_{1}\left|\frac{x}{L_{1}}\right|^{p}+\epsilon_{2}\left|\frac{y}{L_{2}}\right|^{l}+\epsilon_{3}\left|\frac{z}{L_{3}}\right|^{q}
$$

where $L_{1}, L_{2}$ and $L_{3}$ are the linear sizes of the volume, and $\varepsilon_{1}, \varepsilon_{2}, \varepsilon_{3}, p, l$ and $q$ the parameters, Eq. (21) yields

$$
N=z \lambda^{-3} \chi I(p, l, q) \beta^{-\eta+1 / 2},
$$

with

$$
\chi=\frac{L_{1} L_{2} L_{3}}{\epsilon_{1}^{1 / p} \epsilon_{2}^{1 / l} \epsilon_{3}^{1 / q}}, \quad I(p, l, q)=\frac{8}{p l q} \Gamma(1 / p) \Gamma(1 / l) \Gamma(1 / q), \quad \eta=\frac{1}{p}+\frac{1}{l}+\frac{1}{q}+\frac{1}{2} .
$$

In the derivation we have used the formula for the gamma function 


$$
\Gamma(z)=\int_{0}^{\infty} t^{z-1} e^{-t} d t
$$

Relation (5) determines the chemical potential $\mu$ from a given total number $N$ of atoms. The total kinetic energy can be obtained as

$$
K_{\mathrm{MB}}=\int \frac{p^{2}}{2 m} d N(\mathbf{r}, \mathbf{p}, T)=\frac{3}{2} k T \frac{z}{\lambda^{3}} \int \exp [-\beta V(\mathbf{r})] d \mathbf{r}=\frac{3}{2} N k T,
$$

which is just a result of the energy equipartition principle. Similarly, the total potential energy is

$$
V_{\mathrm{MB}}=\int V(\mathbf{r}) d N(\mathbf{r}, \mathbf{p}, T)=\left(\frac{1}{p}+\frac{1}{l}+\frac{1}{q}\right) \frac{z \chi I(p, l, q)}{\lambda^{3} \beta^{\eta+1 / 2}} .
$$

Thus, the total energy of the trapped ideal Maxwell-Boltzmann gas is

$$
E_{\mathrm{MB}}^{t}=(\eta+1) N k T \text {. }
$$

We next consider an ideal Bose-Einstein gas in the generic power law potential. Under the semi-classcial approximation [1],6, 6] the local number density is given by the Bose-Einstein distribution function

$$
n_{\mathrm{BE}}(\mathbf{r}, \mathbf{p}, T)=\left\{\exp \left[\beta\left(p^{2} / 2 m+V(\mathbf{r})-\mu\right)\right]-1\right\}^{-1},
$$

from which the reduced spatial distribution of atoms is [6,11]

$$
\rho(\mathbf{r})=\lambda^{-3} g_{3 / 2}[\exp (-\beta(V(\mathbf{r})-\mu))],
$$

where $g_{\nu}(x)=\sum_{j=1} x^{j} / j^{\nu}$.

The critical temperature for an ideal Bose-Einstein gas in the generic power law potential to undergo BEC has been found in Refs. [6,8] to be

$$
T_{c}=\frac{1}{k}\left(\frac{N\left(2 \pi \hbar^{2}\right)^{3 / 2}}{m^{3 / 2} \zeta(\eta+1) \chi I(p, l, q)}\right)^{1 /(\eta+1)},
$$

where $\zeta(\nu)=g_{\nu}(1)$ is the Riemann zeta function.

The cases of the temperature below and above $T_{c}$ should be treated separately. For $T>T_{c}$, integrating the number density function (11) over the phase space, we obtain the total number of atoms [9]

$$
N=h^{-3} \int n_{\mathrm{BE}}(\mathbf{r}, \mathbf{p}, T) d \mathbf{r} d \mathbf{p}=\frac{\chi I(p, l, q)}{\lambda^{3} \beta^{\eta-1 / 2}} g_{\eta+1}(z) .
$$

In the derivation we have used the definition of the function $g_{\nu}$. Similarly, we have the total kinetic energy

$$
K_{\mathrm{BE}}=h^{-3} \int \frac{p^{2}}{2 m} n_{\mathrm{BE}}(\mathbf{r}, \mathbf{p}, T) d \mathbf{p} d \mathbf{r}=\frac{3}{2} \frac{\chi I(p, l, q)}{\lambda^{3} \beta^{\eta+1 / 2}} g_{\eta+2}(z),
$$

and the total potential energy

$$
V_{\mathrm{BE}}=\int V(\mathbf{r}) \rho(\mathbf{r}) d \mathbf{r}=\frac{\chi I(p, l, q)}{\lambda^{3} \beta^{\eta+1 / 2}}\left(\frac{1}{p}+\frac{1}{l}+\frac{1}{q}\right) g_{\eta+2}(z) .
$$

The total energy of the trapped ideal Bose gas is then [12]

$$
E_{\mathrm{BE}}^{t}=\frac{g_{\eta+2}(z)}{g_{\eta+1}(z)}(\eta+1) N k T
$$

Comparing with the trapped ideal Maxwell-Boltzmann gas, we see that the only difference in the two total energy expressions is the factor $g_{\eta+2}(z) / g_{\eta+1}(z)$ for the Bose gas, which breaks the linear dependence of the total energy on the temperature.

When the temperature is below $T_{c}$, the contribution to the energy of the system comes only from the normal component of the Bose gas. From Eqs. (14), (15), (16) and (17) the kinetic, potential and total energy of the system can be written as 


$$
\begin{aligned}
K_{\mathrm{BE}} & =\frac{3}{2} \frac{g_{\eta+2}(1)}{g_{\eta+1}(1)}\left(\frac{T}{T_{c}}\right)^{\eta+1} N k T, \\
V_{\mathrm{BE}} & =\left(\frac{1}{p}+\frac{1}{l}+\frac{1}{q}\right) \frac{g_{\eta+2}(1)}{g_{\eta+1}(1)}\left(\frac{T}{T_{c}}\right)^{\eta+1} N k T, \\
E_{\mathrm{BE}}^{t} & =(\eta+1) \frac{g_{\eta+2}(1)}{g_{\eta+1}(1)}\left(\frac{T}{T_{c}}\right)^{\eta+1} N k T .
\end{aligned}
$$

The energy of the system will go to zero when the temperature approaches zero.

So far we have not considered the role of the mutual interaction of Bose atoms. For a trapped interacting Bose gas, we divide the total energy into three parts: the kinetic energy $K_{i n t}$, potential energy $V_{\text {int }}$ and interaction energy $U_{\text {int }}$, i.e.

$$
E_{i n t}^{t}=K_{i n t}+V_{i n t}+U_{i n t},
$$

In the local density approximation or semi-classical approximation, the local number density function of a trapped interacting Bose gas is given by [7] 11$]$

$$
n(\mathbf{r}, \mathbf{p}, T)=\left\{\exp \left[\beta\left(p^{2} / 2 m+V(\mathbf{r})+4 a \lambda^{2} \rho(\mathbf{r})-\mu\right)\right]-1\right\}^{-1} .
$$

In similarity to the non-interacting Bose gas, the cases of the temperature above and below $\tilde{T}_{c}$ need to be dealt with separately, where $\tilde{T}_{c}$ is the critical temperature of the trapped interacting Bose gas [8]. At a temperature above $\tilde{T}_{c}$, from (22) the total number of atoms is [9]

$$
N=\frac{1}{h^{3}} \int n(\mathbf{r}, \mathbf{p}, T) d \mathbf{p} d \mathbf{r}=\frac{\chi I(p, l, q)}{\lambda^{3} \beta^{\eta-1 / 2}}\left[g_{\eta+1}(z)-\frac{2 a}{\lambda} F_{3 / 2,3 / 2, \eta-1}(z)\right],
$$

where

$$
F_{\delta, \nu, \eta}(x)=\sum_{i, j}^{\infty} \frac{x^{i+j}}{i^{\delta} j^{\nu}(i+j)^{\eta-1 / 2}} .
$$

In the derivation we have expanded the exponential density function with respect to the small parameter $a / \lambda$, and kept only terms up to the lowest order in $a / \lambda$ [8.9]. Up to this first order correction, Eq. (12) can be used. By the same procedure, the total kinetic and potential energy are obtained as

$$
\begin{aligned}
K_{\text {int }} & =\frac{1}{h^{3}} \int \frac{p^{2}}{2 m} n(\mathbf{r}, \mathbf{p}, T) d \mathbf{p} d \mathbf{r}=\frac{3}{2} \frac{\chi I(p, l, q)}{\lambda^{3} \beta^{\eta+1 / 2}}\left[g_{\eta+2}(z)-\frac{4 a}{\lambda} F_{3 / 2,3 / 2, \eta}(z)\right], \\
V_{\text {int }} & =\frac{1}{h^{3}} \int V(\mathbf{r}) n(\mathbf{r}, \mathbf{p}, T) d \mathbf{p} d \mathbf{r}=\left(\frac{1}{p}+\frac{1}{l}+\frac{1}{q}\right) \frac{\chi I(p, l, q)}{\lambda^{3} \beta^{\eta+1 / 2}}\left[g_{\eta+2}(z)-\frac{4 a}{\lambda} F_{1 / 2,3 / 2, \eta+1}(z)\right] .
\end{aligned}
$$

Similarly, with $V(\mathbf{r})$ being replaced by $2 a \lambda^{2} k T \rho(\mathbf{r})$, the interaction energy is calculated as

$$
U_{\text {int }}=\int 2 a \lambda^{2} k T \rho^{2}(\mathbf{r}) d \mathbf{r}=\frac{2 a \chi I(p, l, q)}{\lambda^{4} \beta^{\eta+1 / 2}} F_{3 / 2,3 / 2, \eta}(z) .
$$

From the above three expressions for $K_{i n t}, V_{\text {int }}$ and $U_{\text {int }}$, we see that the correction to the energy of the trapped ideal Bose gas due to the mutual interaction of atoms is proportional to $a / \lambda$, hence is very small for a weak interaction. This explains why the ideal Bose gas result agrees well with the experimental data above $\tilde{T}_{c}$.

When the temperature is below $\tilde{T}_{c}$, a region where a great number of atoms are in the BEC state forms. In the local density approximation, a relation between density and potential inside the region reads [1]

$$
V(\mathbf{r})+4 \pi a \rho(\mathbf{r}) \hbar^{2} / m=V\left(\mathbf{r}_{0}\right)+4 \pi a \rho_{0} \hbar^{2} / m, \quad r<r_{0},
$$

where $r_{0}$ corresponds to the edge of the region. At $r=r_{0}^{-}$, we have $\rho\left(r_{0}^{-}\right)=\rho_{0}=\lambda^{-3} g_{3 / 2}(1)$, which is the critical density of the gaseous phase for the untrapped homogeneous ideal Bose gas at temperature $T$. From the above relation, we find the density of the condensed atoms as the difference of $\rho(\mathbf{r})$ and $\rho_{0}$

$$
\rho_{s}(\mathbf{r})=\rho(\mathbf{r})-\rho_{0}=\frac{m}{4 \pi a \hbar^{2}}\left[V\left(\mathbf{r}_{\mathbf{0}}\right)-V(\mathbf{r})\right], \quad r<r_{0} .
$$


In order to obtain $\rho_{s}$, the potential $V\left(r_{0}\right)$ at the edge of the condensed region needs to be determined. Integrating $\rho_{s}$ over the whole volume, we have the total number of atoms in the condensed state

$$
N_{s}=\frac{m \chi}{4 \pi a \hbar^{2}} \frac{I(p, l, q)}{\Gamma(\eta+3 / 2)} V^{\eta+1 / 2}\left(r_{0}\right),
$$

where the derivation involves the integral

$$
\int_{|x|^{p}+|y|^{l}+|z|^{q} \leq 1}\left(1-|x|^{p}-|y|^{l}-|z|^{q}\right) d x d y d z=\frac{I(p, l, q)}{\Gamma(\eta+3 / 2)} .
$$

At the weak interaction limit we may approximate $N_{s}$ and $\tilde{T}_{c}$ by those for the trapped ideal Bose gas [6]8], i.e.

$$
N_{s} \approx N\left[1-\left(\frac{T}{T_{c}}\right)^{\eta+1}\right], \quad T<T_{c}
$$

where we have written $\tilde{T}_{c}$ as $T_{c}$. From Eqs. (30) and (32) we find

$$
V\left(r_{0}\right)=\left\{\frac{4 \pi a \hbar^{2} \Gamma(\eta+3 / 2)}{m \chi I(p, l, q)}\left[1-\left(\frac{T}{T_{c}}\right)^{\eta+1}\right] N\right\}^{2 /(2 \eta+1)} .
$$

For the condensation region at $T<T_{c}$ there exists another relation [1]

$$
k T \ln z-V(\mathbf{r})=\frac{4 \pi a \hbar^{2}}{m}\left[\rho(\mathbf{r})+\rho_{0}\right], \quad\left(r<r_{0}\right) .
$$

Setting $r=r_{0}^{-}$in this formula, from Eq. (33) we find the chemical potential as a descending function of temperature to be

$$
\mu=\frac{8 \pi a \hbar^{2}}{m} \rho_{0}+\left\{\frac{4 \pi a \hbar^{2} \Gamma(\eta+3 / 2)}{m \chi I(p, l, q)}\left[1-\left(\frac{T}{T_{c}}\right)^{\eta+1}\right] N\right\}^{2 /(2 \eta+1)}, \quad\left(T<T_{c}\right),
$$

which, for a cylindrically symmetric harmonic trapping potential, reduces to

$$
\mu=\frac{8 \pi a \rho_{0} \hbar^{2}}{m}+\frac{\hbar \omega_{0}}{2}\left\{\frac{15 a N}{a_{0}}\left[1-\left(\frac{T}{T_{c}}\right)^{3}\right]\right\}^{2 / 5} \stackrel{T \rightarrow 0}{\longrightarrow} \frac{\hbar \omega_{0}}{2}\left\{\frac{15 a N}{a_{0}}\right\}^{2 / 5}
$$

where $a_{0}$ is the geometric mean characteristic length of the trap, i.e. $a_{0}^{2}=\hbar /\left(m \omega_{0}\right)$ with $\omega_{0}^{3}=\omega_{\perp}^{2} \omega_{z}$. This is consistent with the Ginzburg-Pitaevskii-Gross mean field theory [13].

According to Refs. [11,14, the density of the mutual interaction energy in the condensation region is

$$
\begin{aligned}
u_{i n t} & =2 a \lambda^{2} k T \rho^{2}(\mathbf{r})-a \lambda^{2} k T \rho_{s}^{2}(\mathbf{r}) \\
& =a \lambda^{2} k T\left[2 \rho_{0}^{2}(\mathbf{r})+4 \rho_{0} \rho_{s}(\mathbf{r})+\rho_{s}^{2}(\mathbf{r})\right], \quad\left(r<r_{0}\right),
\end{aligned}
$$

which, after integrating over the condensation region $\left(r<r_{0}\right)$, gives

$$
U_{i n t}=\chi I(p, l, q) V^{\eta+1 / 2}\left(r_{0}\right)\left[\frac{4 \pi a \hbar^{2} \rho_{0}^{2}}{m \Gamma(\eta+1 / 2) V\left(r_{0}\right)}+\frac{2 \rho_{0}}{\Gamma(\eta+3 / 2)}+\frac{m V\left(r_{0}\right)}{4 \pi a \hbar^{2} \Gamma(\eta+5 / 2)}\right] .
$$

The first two terms depending on $\rho_{0}$ vanish when the temperature approaches zero. So, at low temperature the main contribution to $U_{\text {int }}$ comes from the third term corresponding to the interaction within the condensed atoms. We may then neglect the first and second terms of (38) to write

$$
U_{i n t} \approx \frac{1}{\Gamma(\eta+5 / 2)}\left(\frac{4 \pi a \hbar^{2}}{m \chi I(p, l, q)}\right)^{2 /(2 \eta+1)}\left\{\left[1-\left(\frac{T}{T_{c}}\right)^{\eta+1}\right] \Gamma(\eta+3 / 2) N\right\}^{(2 \eta+3) /(2 \eta+1)} .
$$


To an approximation, we may neglect any correction to the energy from the normal component, and express the energy of the trapped interacting Bose gas as that of the trapped ideal Bose gas plus the only correction from the mutual interaction within the condensed component.

In the experiment the trapping potential is [10]

$$
V(\mathbf{r})=\frac{1}{2} m \omega_{\perp}^{2} r_{\perp}^{2}+\frac{1}{2} \omega_{z}^{2} r_{z}^{2}
$$

which is harmonic. In the ideal Bose gas approximation, at a temperature $T$ above $T_{c}$, Eqs. (15) and (16) reduce to

$$
K=\frac{3}{2} \frac{g_{4}(z)}{g_{3}(z)} N k T, \quad V=\frac{3}{2} \frac{g_{4}(z)}{g_{3}(z)} N k T,
$$

where the fugacity $z$ is related to the total number of atoms as

$$
N=\frac{g_{3}(z)}{\hbar^{3} \omega_{\perp}^{2} \omega_{z} \beta^{3}},
$$

which yields $g_{3}(z)=\left(T_{c} / T\right)^{3} g_{3}(1)$. The energy of the atom interaction is negligible at $T>T_{c}$.

When the temperature is below $T_{c}$, from Eqs. (18) and (19), the approximate kinetic and potential energy are

$$
K^{-}=\frac{3}{2} \frac{\zeta(4)}{\zeta(3)}\left(\frac{T}{T_{c}}\right)^{3} N k T, \quad V^{-}=K^{-} .
$$

The contribution to the energy from the interaction within the condensation component is now significant. From Eqs. (13) and (39), this interaction energy is

$$
U^{-}=\frac{1}{7}\left(\frac{15 a}{a_{0}}\right)^{2 / 5} N^{1 / 15}(\zeta(3))^{1 / 3}\left[1-\left(\frac{T}{T_{c}}\right)^{3}\right]^{7 / 5} N k T_{c} .
$$

In the experiment the trapped atoms are ${ }^{87} \mathrm{Rb}[10]$. The $s$-wave scattering length of ${ }^{87} \mathrm{Rb}$ is $a \approx 100$ in the unit of the Bohr radius $a_{B}$. The trapping harmonic potential is axially symmetric with the frequency ratio of axial to radial being $\sqrt{8}$. The final stage of the evaporative cooling is performed at $\nu_{z}=373 \mathrm{~Hz}$. The trapping field is then turned off to initiate the expansion of the atom cloud non-adiabatically. The kinetic energy $E_{\exp }$ of atoms is measured after a sufficient expansion which ensures the interaction energy to convert to purely kinetic energy. Therefore, we may estimate $E_{\exp }$ as the sum of $K^{-}$and $U^{-}$, i.e.

$$
\frac{E_{\text {exp }}}{N k T_{c}}=\frac{3}{2} \frac{g_{4}(z)}{g_{3}(z)}\left(\frac{T}{T_{c}}\right), \quad \text { for } \quad T>T_{c}
$$

and

$$
\frac{E}{N k T_{c}}=\frac{3}{2} \frac{g_{4}(1)}{g_{3}(1)}\left(\frac{T}{T_{c}}\right)^{4}+0.449\left(\frac{a}{a_{0}}\right)^{2 / 5} N^{1 / 15}\left[1-\left(\frac{T}{T_{c}}\right)^{3}\right]^{7 / 5}, \quad \text { for } \quad T<T_{c} .
$$

Assume that the total number of atoms in the trap is 40000. The scaled energy per particle, $E / N k T_{c}$, is plotted versus the scaled temperature, $T / T_{c}$, in Fig. 1, where the solid, dashed and dotted lines correspond to the ideal Bose gas, ideal Maxwell-Boltzmann and interacting Bose gas, respectively. When the temperature is rather high, they are very close. However, at low temperature the differences among them are prominent. The theoretical result of the interacting Bose gas is in qualitative agreement with the experimental data. We have considered only the contribution from the mutual interaction of the condensed atoms. If the finite size effect [15, 16] and all other interaction terms are included, a quantitative comparison with experimental data can be made.

\section{ACKNOWLEDGMENTS}

The authors thank Hao Bai-lin for his encouragement and useful discussions. This work was supported in part by the National Natural Science Foundation of China. 
[1] Kerson Huang, Statistical Mechanics, ( Wiley, New York, 1987), 2nd ed .

[2] O. Penrose and L. Onsager, Phys. Rev. 104, 576 (1956).

[3] M.H. Anderson, J.R. Ensher, M.R. Matthews, C.E. Wieman, and E.A. Cornell, Science 269, 198 (1995).

[4] C.C. Bradley, C.A. Sackett, J.J. Tollett, and R.G. Hulet, Phys. Rev. Lett. 75, 1687 (1995).

[5] K.B. Davis, M.-O. Mewes, M.R. Andrew, N.J. van Druten, D.S. Durfee, D.M. Kurn, and W. Ketterle, Phys. Rev. Lett. 75, 3969 (1995).

[6] Vanderlei Bagnato, David E. Pritchard, and Daniel Kleppner, Phys. Rev. A35, 4354 (1987).

[7] S. Giorgini, L. Pitaevskii, and S. Stringari, Phys. Rev. A54, 4633 (1996).

[8] Hualin Shi, Wei-mou Zheng, cond-mat/9609241.

[9] Hualin Shi, Wei-mou Zheng, cond-mat/9611171 .

[10] J.R. Ensher, D.S. Jin, M.R. Matthews, C.E. Wieman, and E.A. Cornell, Phys. Rev. Lett. 4984 (1996) .

[11] T.T. Chou, C.N. Yang, and L.W. Yu, Phys. Rev. A53, 4257 (1996).

[12] P.W.H. Pinkse, A.Mosk, M. Weidemüller, M.W. Reynolds, T.W. Hijmans, and J.T.M. Walraven, physics/9611026 .

[13] G. Baym, and C.J. Pethick, Phys. Rev. Lett. 76, 6 (1996).

[14] Kerson Huang and C.N. Yang, Phys. Rev. 105, 776 (1957).

[15] S. Grossmann and M. Holthaus, phys. Lett. A208, 188 (1995).

[16] W. Ketterle and N.J. van Druten, Phys. Rev. A54, 656 (1996).

\section{Figure Captions}

Fig. 1 Plot of the scaled energy per particle $E / N k T_{c}$ of trapped gas vs. scaled temperature $T / T_{c}$. The solid, dashed and dotted lines correspond to an ideal Bose gas, ideal Maxwell-Boltzmann gas and interacting Bose gas, respectively. 


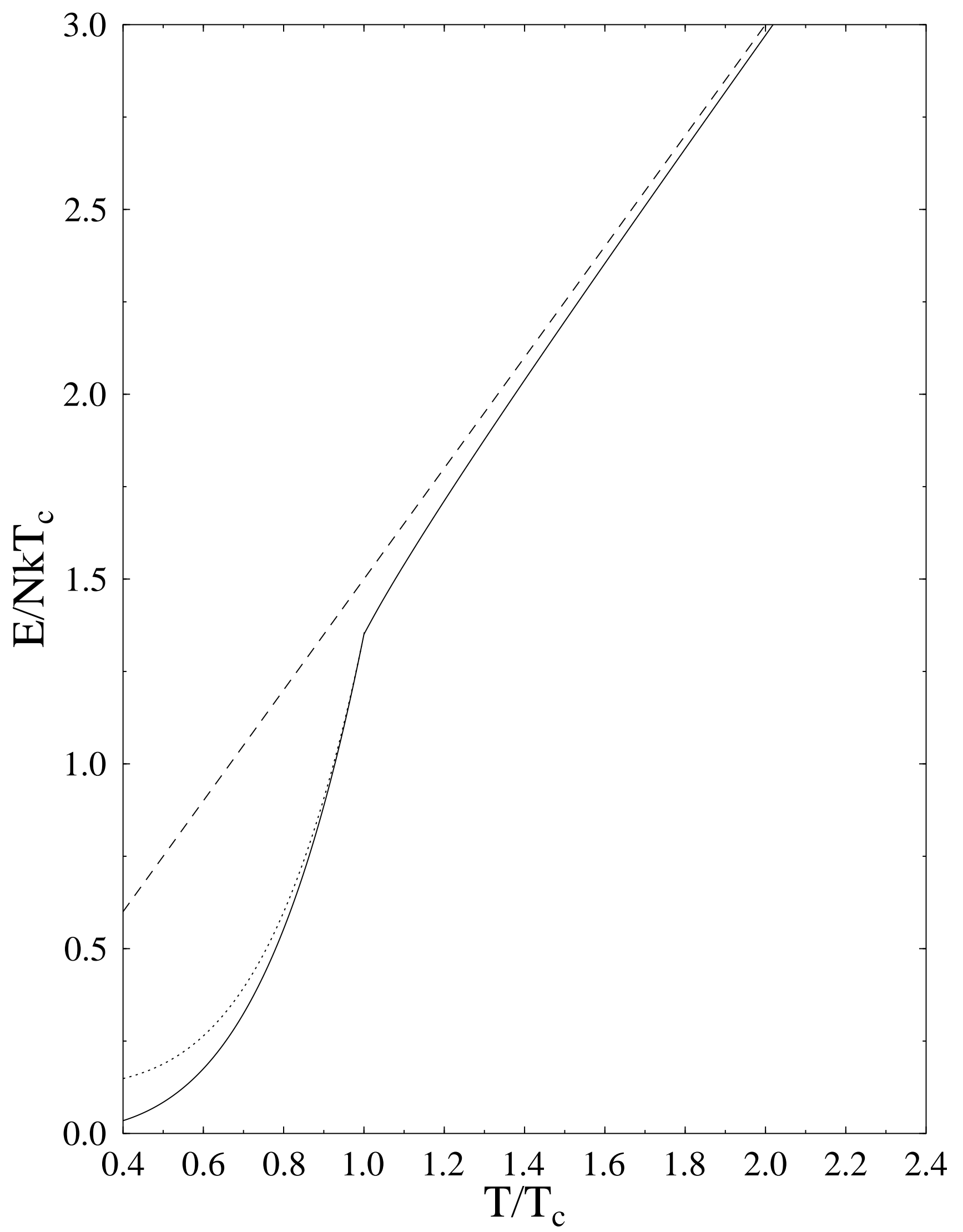

